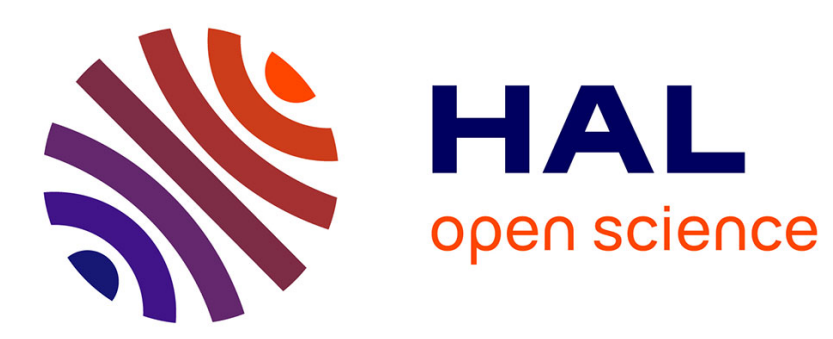

\title{
On Using Norm Estimators for Event-Triggered Control with Dynamic Output Feedback
}

Aneel Tanwani, Andrew Teel, Christophe Prieur

\section{To cite this version:}

Aneel Tanwani, Andrew Teel, Christophe Prieur. On Using Norm Estimators for Event-Triggered Control with Dynamic Output Feedback. CDC 2015 - 54th IEEE Conference on Decision and Control, Dec 2015, Osaka, Japan. 10.1109/CDC.2015.7403081 . hal-01238648

\section{HAL Id: hal-01238648 \\ https://hal.science/hal-01238648}

Submitted on 6 Dec 2015

HAL is a multi-disciplinary open access archive for the deposit and dissemination of scientific research documents, whether they are published or not. The documents may come from teaching and research institutions in France or abroad, or from public or private research centers.
L'archive ouverte pluridisciplinaire HAL, est destinée au dépôt et à la diffusion de documents scientifiques de niveau recherche, publiés ou non, émanant des établissements d'enseignement et de recherche français ou étrangers, des laboratoires publics ou privés. 


\section{On Using Norm Estimators for Event-Triggered Control with Dynamic Output Feedback}

Aneel Tanwani
Andrew Teel

\author{
Christophe Prieur
}

\begin{abstract}
For feedback stabilization of a control system using dynamic output feedback, we consider the problem of finding two different sequences of time instants at which the sampled outputs (respectively, control inputs) must be sent to the controller (resp. the plant). Instead of static inequalities, the states of so-called norm estimators are used to determine sampling instants. Using the tools from Lyapunov theory for hybrid systems and stability of cascaded nonlinear systems, it is shown that the closed loop system is globally asymptotically stable. Additional assumptions are required on the controller and system dynamics to guarantee that the proposed sampling routines do not lead to an accumulation of sampling times over a finite interval.
\end{abstract}

\section{INTRODUCTION}

Sampled data control of continuous-time dynamical systems has been a topic of central interest in control community for a long time. Some of the earlier works were based on constructing discrete-time approximations of the nonlinear dynamics, see [15] and the references therein. In the later works, it is shown that if a stabilizing controller exists with certain robustness properties, then bounds on sampling periods for the measurements can be obtained which would preserve asymptotic stability of the closed-loop system [16], hence control with periodic sampling is achieved.

Over the past few years, however, event-triggered techniques have regained interest [4], where the measurements are not sent periodically to the controller, but instead the sampling times are determined based on the current value of the state. A recent article [12] provides a nice tutorial exposition into the subject, and sums up most of the work done so far. A common framework for event-triggered control involves a stabilizing feedback controller and a triggering mechanism that determines when to send the updated measurements to the controller. While the feedback control is usually available "off-the-shelf," different strategies and variants are adopted for triggering mechanism depending upon the particular problem setup. Initial approaches for event-triggering mechanism involve keeping the difference between current value of the state and the last updated measurement relatively small [3], [14], [23]. Another technique is to monitor the derivative of the Lyapunov function associated with the closed-loop system, and if it starts approaching zero, then we update the measurement knowing that the new measurement will make the derivative sufficiently negative [17], [19].

A. Tanwani is with Department of Mathematics, University of Kaiserslautern, Germany. A. Teel is with University of California at Santa Barbara, USA. C. Prieur is with Gipsa-Lab (CNRS), Grenoble, France. This work has been partially supported by the LabEx PERSYVAL-Lab (ANR-11-LABX0025-01) and by the ANR project LimICoS contract number 12-BS03-00501. Corresponding author: tanwani@mathematik. uni-kl.de.
In the references cited above, the triggering mechanisms are based on using the full-state measurements and when it comes to using output (partial state) measurements for feedback, rather than full-state feedback, then relatively little has been done. If we directly generalize the techniques based on keeping the error (between the last sampled output and the current value of the output) small, then such methods lead to Zeno phenomenon, where we need to send infinitely many samples in finite-time and hence the technique is not feasible for implementation in practice. Some refinements have been proposed in [6], [13], [24], where instead of asymptotic stabilization, the authors modify the event function to achieve practical stabilization so that the trajectories of the closedloop system only converge to a ball defined by a design parameter. Unsurprisingly, that parameter also determines the minimum inter-sampling time as well.

Asymptotic stability with output-feedback and eventtriggered sampling has also been considered in more recent works where a certain dwell-time is enforced between two consecutive sample updates to overcome Zeno phenomenon. Recently introduced notion of periodic event-triggered control could be seen as an implementation of this idea [10], [11] where it is assumed that the continuous-time plant is already discretized with some fixed sampling-rate, or a certain sampling period is precalculated to asymptotically stabilize the system. One then focuses on adding another level of sampling strategy (which is event-triggered) that would reduce the sampling rate for measurements even further. The results appearing in [11] for linear systems take disturbances into account, and derive minimum intersampling time for full-state feedback case only. The idea of forcing a certain dwell-time between two consecutive sample-updates has also been adopted in nonlinear setting for output feedback laws in [1].

The work discussed so far involves formulating the even function in terms of the measured system variable, be it state or output. More recently, we have seen dynamic event generators where an auxiliary first order dynamical system is constructed to determine the sampling instants [5], [7]. It has been shown that adding dynamics results in increased inter-sampling time compared to the static event-triggering conditions. However, these works only discuss stabilization with full state static feedback control laws; sampled-data feedback with dynamic event-triggering using partial state measurements, or output feedback has remain unaddressed.

In this paper, we consider the problem of implementing observer-based dynamic output feedback controller for asymptotic stabilization of nonlinear systems using event- 


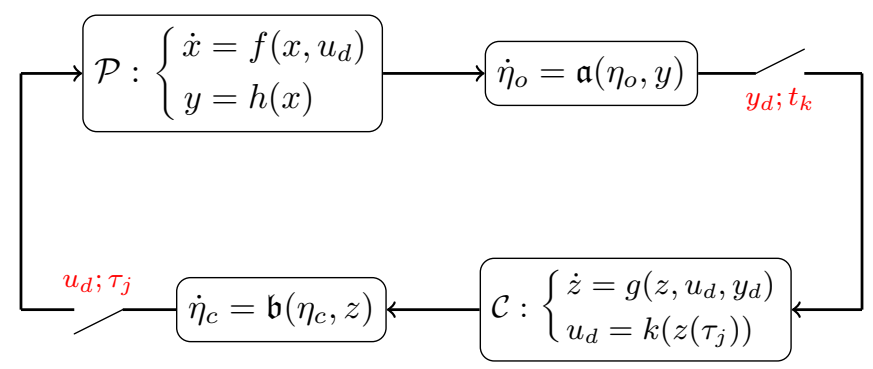

Fig. 1. Feedback loop where the inputs and outputs are time-sampled and the sampling instants are determined by the dynamic filters $\eta_{c}$ and $\eta_{o}$, respectively.

triggered sampling with norm estimators, or dynamic event generators. Another novel aspect of our approach lies in designing separate first order dynamic filters to determine sampling instants for control inputs and outputs. Just like the approach adopted in the state-feedback case, our approach is also based on keeping the error between the current value of the output and the last output sample small, and the difference between the actual output and sampled output is compared with a certain function of the state of the norm estimator. The sampling times for control inputs are generated by an event-triggering rule that depends upon the state of the controller. In doing so, we do not rely on precalculating some fixed sampling period between two output/input sampling times. The controller, using these sampled outputs, is assumed to be designed based on the principle of certainty equivalence, that is, an estimate of the current state is first computed, which is in turn fed into the control law. A graphical illustration of the control architecture that we want to realize is given in Figure 1, in which the dynamics for $\eta_{o}$ and $\eta_{c}$ are to be designed, and the sampling times $t_{k}$ for outputs, and $\tau_{j}$ for inputs, are determined from the real-time values of $\eta_{o}$ and $\eta_{c}$.

The framework of hybrid system [9] is used to describe the overall closed-loop system and sufficient conditions are given for asymptotic stability of this system. We develop these intuitive ideas using Lyapunov-based analysis to show that the closed-loop system is indeed stable, and that the major obstacle of the accumulation of sampling times is also removed. To show that our sampling algorithms are feasible for implementation, we are able to show that the output and input sampling times do not accumulate over a finite interval. A property of the proposed sampling routines is that the sampling times of the output and control input are not necessarily synchronized.

\section{Problem Setup}

We consider nonlinear dynamical systems of the form

$$
\begin{aligned}
& \dot{x}=f(x, u) \\
& y=h(x)
\end{aligned}
$$

where $x, u, y$ denote the state trajectory, input and the output respectively. For stabilization of system (1), we choose to work with the following class of controllers:

$$
\begin{aligned}
\dot{z} & =g(z, u, y) \\
u & =k(z) .
\end{aligned}
$$

In our approach, the dynamical system (2a) plays the role of state estimator, and the control input $u$ is some function of the estimated state variable. The problem of stabilization of nonlinear systems with dynamic output feedback is wellstudied in the literature, see [2] for a survey, or [25] for various tools developed for solving this problem. In this article, we are interested in generalizing output feedback controllers of the form (2) when only the time-sampled output measurements are available to the controller, and the input $u$ sent to the plant is also time-sampled. To address this problem, we first layout some basic hypotheses on system and controller data (2) that will be used later on.

\section{A. Nominal Output Feedback}

We now list certain basic assumptions on system (1) and the controller (2) which relate to robust (with respect to measurement errors) asymptotic stabilization of the closedloop system.

(H1) The vector fields $f: \mathbb{R}^{n} \times \mathbb{R}^{m} \rightarrow \mathbb{R}^{n}$ and $g: \mathbb{R}^{n} \times \mathbb{R}^{m} \times$ $\mathbb{R}^{p} \rightarrow \mathbb{R}^{n}$ are continuous in each of their arguments. The function $h: \mathbb{R}^{n} \rightarrow \mathbb{R}^{p}$ is continuous and there exists a class $\mathcal{K}$ function $\alpha_{h}$ such that

$$
|h(x)| \leq \alpha_{h}(|x|)
$$

(H2) An ISS state estimator: There exist a continuously differentiable function $V_{o}: \mathbb{R}^{n} \rightarrow \mathbb{R}_{>0}$, and functions $\alpha_{o}, \underline{\alpha}_{o}, \bar{\alpha}_{o}, \gamma_{o}$ of class $\mathcal{K}_{\infty}$, which satisfy the following inequalities:

$\underline{\alpha}_{o}(|e|) \leq V_{o}(e) \leq \bar{\alpha}_{o}(|e|)$
$\left\langle\nabla V_{o}, f(x, u)-g\left(z, u, y+d_{y}\right)\right\rangle \leq-\alpha_{o}\left(V_{o}(e)\right)+\gamma_{o}\left(\left|d_{y}\right|\right)$

where $e:=x-z$ denotes the state estimation error.

(H3) An ISS control law: There exist a continuously differentiable function $V_{c}: \mathbb{R}^{n} \rightarrow \mathbb{R}_{\geq 0}$, functions $\alpha_{c}, \underline{\alpha}_{c}, \bar{\alpha}_{c}, \gamma_{c}$ of class $\mathcal{K}_{\infty}$, and a state feedback control law $k: \mathbb{R}^{n} \rightarrow \mathbb{R}^{m}$ such that

$$
\begin{gathered}
\underline{\alpha}_{c}(|x|) \leq V_{c}(x) \leq \bar{\alpha}_{c}(|x|) \\
\left\langle\nabla V_{c}, f\left(x, k\left(x+d_{e}\right)\right)\right\rangle \leq-\alpha_{c}\left(V_{c}(x)\right)+\gamma_{c}\left(\frac{\left|d_{e}\right|}{2}\right) .
\end{gathered}
$$

(H4) As $r \rightarrow 0^{+}$, we have $\left(\gamma_{c} \circ \underline{\alpha}_{o}^{-1}\right)(r)=O\left(\alpha_{o}(r)\right)$, that is, there exists a constant $M>0$ such that

$$
\lim _{r \rightarrow 0^{+}} \frac{\left(\gamma_{c} \circ \underline{\alpha}_{o}^{-1}\right)(r)}{\alpha_{o}(r)} \leq M
$$

Remark 1 (Design methodology): Hypotheses (H2) and (H3) allow us to decompose the problem of dynamic of output feedback into two components: first is to design a state estimator, and then apply a static control law which is robust with respect to measurement errors. Designing control 
laws which are ISS with respect to measurements of state variable has remained a topic of major interest in the control community and several techniques now exist depending on the system class. The state estimators, that one typically designs for a nonlinear system (using high-gain, or passivity approach), are robust with respect to output measurement error but the estimate of the form (3b) is typically not stated in such works. We refer the reader to a recent paper [20] which deals with designing estimators of this form.

Remark 2 (Why (H4)?): We will use (H4) in the proof of stability of closed-loop system which requires the sum of $V_{c}$ and a $\mathcal{K}_{\infty}$ function of $V_{o}$ as the Lyapunov function. This construction is inspired by [21] and it allows us to invoke arguments related to the stability of the cascaded nonlinear systems. If the functions $\underline{\alpha}_{c}$ and $\gamma_{c}$ are quadratic, and $\alpha_{o}$ is linear, as one would usually obtain in the linear case with quadratic $V_{o}$ and $V_{c}$, then (H4) is satisfied.

\section{SAMPling Algorithms}

We will now use the controller structure given in the previous section to study the problem of output feedback stabilization with sampled outputs and inputs. To design sampling algorithms, we consider the following auxiliary dynamical system:

$$
\dot{\eta}_{o}:=-\beta_{o}\left(\eta_{o}\right)+\rho_{o}(|y(t)|)+\gamma_{o}\left(\left|y(t)-y\left(t_{k}\right)\right|\right)
$$

and on the controller side

$$
\dot{\eta}_{c}:=-\beta_{c}\left(\eta_{c}\right)+\rho_{c}\left(\frac{|z(t)|}{2}\right)+\gamma_{c}\left(\left|z(t)-z\left(\tau_{j}\right)\right|\right)
$$

with initial conditions $\eta_{o}(0)>0$, and $\eta_{c}(0)>0$. In the above equations, $\beta_{o}, \rho_{o}, \rho_{c}$, and $\beta_{c}$ are all functions of class $\mathcal{K}$, which would be specified later. One may notice that, if $\beta_{o}(r)=\alpha_{o}(r)$ and $\beta_{c}(r)=\alpha_{c}(r)$ are linear, then in the light of (H2) and (H3), the dynamic filters in (6a) and (6b) play the role of norm estimators [22] for error dynamics $e=x-z$, and the closed-loop dynamics for the state $x$, respectively.

We use the sample-and-hold strategy for sampling, that is, the outputs and inputs are updated at certain discrete times, and in-between updates, they are held constant. The algorithms that determine the sampling instants for inputs and outputs, can now be defined as a function of $\eta_{o}, \eta_{c}$ given by (6).

a) Output Sampling Rule: It is assumed that the output sent to the controller is updated at time instants $t_{k}, k \in \mathbb{N}$ which is defined inductively as:

$$
t_{k+1}:=\inf \left\{t_{k}<t:\left|y(t)-y\left(t_{k}\right)\right| \geq \sigma_{o}\left(\eta_{o}(t)\right)\right\}
$$

where $\sigma_{o}: \mathbb{R}_{\geq 0} \rightarrow \mathbb{R}_{\geq 0}$ is some positive definite, nondecreasing function to be specified later.

b) Input Sampling Rule: The control input $u(\cdot)$ is updated at time instants $\tau_{j}, j \in \mathbb{N}$, according to the following rule:

$$
\tau_{j+1}:=\inf \left\{\tau_{j}<t:\left|z(t)-z\left(\tau_{j}\right)\right| \geq \sigma_{c}\left(\eta_{c}(t)\right)\right\},
$$

where $\sigma_{c}: \mathbb{R}_{\geq 0} \rightarrow \mathbb{R}_{\geq 0}$ is a positive definite and nondecreasing function which will be specified later.

\section{Stability Analysis}

Using the sampling algorithms from the previous section, the dynamics of the closed-loop system are now written in the framework of hybrid systems [9], where we specify the continuous and discrete dynamics, along with their respective domains. We then invoke tools from the literature related to the stability of such systems to show that for certain choice of the design parameters in (6) and appropriately chosen functions $\sigma_{o}, \sigma_{c}$ in (7), (8), the origin of the closed-loop system is asymptotically stable.

\section{A. Hybrid model of the system}

Using $y_{d}$ and $z_{d}$ to denote the sampled output and sampled controller state, respectively, we can let $\bar{x}:=$ $\left(x, z, \eta_{c}, \eta_{o}, y_{d}, z_{d}\right) \in \mathbb{R}^{\bar{n}}$, where $\bar{n}=3 n+p+2$, is the augmented state variable for the closed-loop system. The flow set $\mathcal{C}$ for the state variables (where they all satisfy a certain ordinary differential equation) is defined as

$$
\mathcal{C}:=\mathcal{C}_{o} \cap \mathcal{C}_{c} \cap \mathcal{C}_{\eta}
$$

where we define

$$
\begin{gathered}
\mathcal{C}_{o}:=\left\{\bar{x}:\left|h(x)-y_{d}\right| \leq \sigma_{o}\left(\eta_{o}\right)\right\}, \\
\mathcal{C}_{c}:=\left\{\bar{x}:\left|z-z_{d}\right| \leq \sigma_{c}\left(\eta_{c}\right)\right\}, \\
\mathcal{C}_{\eta}:=\left\{\bar{x}: \eta_{o} \geq 0 \wedge \eta_{c} \geq 0\right\} .
\end{gathered}
$$

The jump set $\mathcal{D}$ where the state variables may get reset is given by:

$$
\mathcal{D}:=\mathcal{D}_{c} \cup \mathcal{D}_{o}
$$

where

$$
\begin{gathered}
\mathcal{D}_{o}:=\left\{\bar{x}:\left|h(x)-y_{d}\right| \geq \sigma_{o}\left(\eta_{o}\right)\right\} \\
\mathcal{D}_{c}:=\left\{\bar{x}:\left|z-z_{d}\right| \geq \sigma_{c}\left(\eta_{c}\right)\right\} .
\end{gathered}
$$

Clearly, the sets $\mathcal{C}$ and $\mathcal{D}$ are closed. By construction, the jump set $\mathcal{D}$ for the closed-loop hybrid system also allows for two jumps simultaneously, that is, $y_{d}$ and $z_{d}$ may get updated at the same time instant. The corresponding set of differential and difference equation on these sets are:

$$
\bar{x} \in \mathcal{C}: \begin{cases}\dot{x} & =f\left(x, k\left(z_{d}\right)\right) \\ \dot{z} & =g\left(z, k\left(z_{d}\right), y_{d}\right) \\ \dot{z}_{d} & =0 \\ \dot{y}_{d} & =0 \\ \dot{\eta}_{o} & =-\beta_{o}\left(\eta_{o}\right)+\rho_{o}(|h(x)|)+\gamma_{o}\left(\left|h(x)-y_{d}\right|\right) \\ \dot{\eta}_{c} & =-\beta_{c}\left(\eta_{c}\right)+\rho_{c}\left(\frac{|z|}{2}\right)+\gamma_{c}\left(\left|z-z_{d}\right|\right)\end{cases}
$$

$\bar{x} \in \mathcal{D}_{c}:\left\{z_{d}^{+}=z\right.$

$\bar{x} \in \mathcal{D}_{o}:\left\{y_{d}^{+}=h(x)\right.$.

Proposition 1: The closed-loop system (11) satisfies the basic assumptions listed in [9, Assumption 6.5], and is, hence, nominally well-posed.

\section{B. Design of sampling functions}

The choice of functions $\sigma_{o}, \sigma_{c}$ depends on the construction of a function $q$ which will also be used to define the 
Lyapunov function of system (11). Under assumption (H4), it is possible to introduce a continuous nondecreasing function $q: \mathbb{R}_{\geq 0} \rightarrow \mathbb{R}_{\geq 0}$ that satisfies [21, Lemma 2]:

$$
q(s) \geq \frac{4\left(\gamma_{c} \circ \underline{\alpha}_{o}^{-1}\right)(s)}{\alpha_{o}(s)} .
$$

The functions $\beta_{o}, \beta_{c}, \sigma_{o}, \sigma_{c}, \rho_{o}$ and $\rho_{c}$ under consideration should satisfy the following design criteria for the stability result to follow:

(D1) Let $\beta_{o}$ and $\beta_{c}$ two smooth functions of class $\mathcal{K}$;

(D2) Let $\theta$ be a function of class $\mathcal{K}_{\infty}$ defined as

$$
\theta_{o}(r):=\alpha_{o}^{-1}\left(2 \gamma_{o}(r)\right)
$$

Choose the functions $\sigma_{o}$ and $\sigma_{c}$ in (9) and (10) such that for some $\varepsilon \in(0,1)$, and for each $s \geq 0$ :

$$
\begin{gathered}
\left(\gamma_{o} \circ \sigma_{o}\right)(s) \cdot\left[1+\left(q \circ \theta_{o} \circ \sigma_{o}\right)(s)\right] \leq(1-\varepsilon) \beta_{o}(s) \\
2\left(\gamma_{c} \circ \sigma_{c}\right)(s) \leq(1-\varepsilon) \beta_{c}(s) .
\end{gathered}
$$

(D3) The functions $\rho_{o}$ and $\rho_{c}$ in (11) are chosen such that for each $s \geq 0$ :

$$
\begin{gathered}
0 \leq\left(\rho_{o} \circ \alpha_{h} \circ \underline{\alpha}_{c}^{-1}\right)(s) \leq(1-2 \varepsilon) \alpha_{c}(s), \\
0 \leq \rho_{c}(s) \leq \min \left\{(1-\varepsilon) \gamma_{c}(s), \varepsilon \alpha_{c}\left(\underline{\alpha}_{c}(s)\right)\right\} .
\end{gathered}
$$

\section{Stability result}

Theorem 1: Consider the closed-loop system (11) under the hypotheses (H1), (H2), (H3), and (H4). If the functions $\beta_{o}, \beta_{c}, \rho_{o}, \rho_{c}$ in (11a) and the functions $\sigma_{o}, \sigma_{c}$ in (9c) are chosen to meet the design criteria (D1), (D2), and (D3), then the origin $\{0\} \in \mathbb{R}^{\bar{n}}$ is globally asymptotically stable (GAS) for the closed-loop system (11).

Proof: We consider the candidate Lyapunov function to be

$$
V:=l\left(V_{o}(e)\right)+V_{c}(x)+\eta_{o}+\eta_{c}
$$

where $e=x-z$, and $l: \mathbb{R}_{\geq 0} \rightarrow \mathbb{R}_{\geq 0}$ is defined as

$$
l(s):=\int_{0}^{s} q(r) d r
$$

Since $q$ is a continuous nondecreasing function, it follows that $l(\cdot)$ is a continuously differentiable function of class $\mathcal{K}_{\infty}$. We now compute a bound on the derivative of each term on the right-hand side of (12) when the state $\bar{x} \in \mathcal{C}$. By letting $y_{d}=y+y_{d}-y$ in the $\dot{z}$ equation, we get

$$
\begin{aligned}
\widehat{l\left(V_{o}(e)\right)} & \leq q\left(V_{o}(e)\right)\left(-\alpha_{o}\left(V_{o}(e)\right)+\gamma_{o}\left(\left|y-y_{d}\right|\right)\right) \\
& \leq q\left(V_{o}(e)\right)\left[-\alpha_{o}\left(V_{o}(e)\right)+\gamma_{o}\left(\sigma_{o}\left(\eta_{o}\right)\right)\right] \\
& \leq-\frac{1}{2} q\left(V_{o}(e)\right) \alpha_{o}\left(V_{o}(e)\right)+q\left(\theta\left(\sigma_{o}\left(\eta_{o}\right)\right)\right) \gamma_{o}\left(\sigma_{o}\left(\eta_{o}\right)\right)
\end{aligned}
$$

where to derive the last inequality, we first consider the case where $\gamma_{o}\left(\sigma_{o}\left(\eta_{o}\right)\right) \leq \frac{1}{2} \alpha_{o}\left(V_{o}(e)\right)$, and then in the second case

$$
\begin{aligned}
\frac{1}{2} \alpha_{o}\left(V_{o}(e)\right) \leq \gamma_{o}\left(\sigma_{o}\left(\eta_{o}\right)\right) \Leftrightarrow V_{o}(e) & \leq \alpha_{o}^{-1}\left(2 \gamma_{o}\left(\sigma_{o}\left(\eta_{o}\right)\right)\right) \\
& =\theta\left(\sigma_{o}\left(\eta_{o}\right)\right)
\end{aligned}
$$

so that $q\left(V_{o}(e)\right) \leq q\left(\theta_{o}\left(\sigma_{o}\left(\eta_{o}\right)\right)\right)$ because $q$ is by construc- tion nondecreasing. Next, from the description of the set $\mathcal{C}$, and using (H1), one obtains

$$
\dot{\eta}_{o} \leq-\beta_{o}\left(\eta_{o}\right)+\gamma_{o}\left(\sigma_{o}\left(\eta_{o}\right)\right)+\rho_{o}\left(\alpha_{h}\left(\underline{\alpha}_{c}^{-1}\left(V_{c}(x)\right)\right)\right)
$$

and similarly by observing that

$$
\rho_{c}\left(\frac{|z|}{2}\right) \leq \rho_{c}\left(\frac{|x|+|e|}{2}\right) \leq \rho_{c}(|x|)+\rho_{c}(|e|)
$$

we get

$$
\begin{aligned}
\dot{\eta}_{c} \leq-\beta_{c}\left(\eta_{c}\right)+\gamma_{c}\left(\sigma_{c}\left(\eta_{c}\right)\right)+\rho_{c}\left(\underline{\alpha}_{c}^{-1}(\right. & \left.\left.V_{c}(x)\right)\right) \\
& +\rho_{c}\left(\underline{\alpha}_{o}^{-1}\left(V_{o}(e)\right)\right) .
\end{aligned}
$$

For computing $\dot{V}_{c}(x)$, we rewrite $\dot{x}$ as

$$
\dot{x}=f\left(x, k\left(x+z-x+z_{d}-z\right)\right)
$$

to obtain

$$
\begin{aligned}
\dot{V}_{c}(x) & \leq-\alpha_{c}\left(V_{c}(x)\right)+\gamma_{c}(|e|)+\gamma_{c}\left(\left|z-z_{d}\right|\right) \\
& \leq-\alpha_{c}\left(V_{c}(x)\right)+\gamma_{c}\left(\underline{\alpha}_{o}^{-1}\left(V_{o}(e)\right)\right)+\gamma_{c}\left(\sigma_{c}\left(\eta_{c}\right)\right)
\end{aligned}
$$

Substituting these bounds in (12) and invoking the conditions imposed by design in (D2) and (D3), we obtain

$\dot{V} \leq-\varepsilon\left[\gamma_{c}\left(\underline{\alpha}_{o}^{-1}\left(V_{o}(e)\right)\right)+\alpha_{c}\left(V_{c}(x)\right)+\beta_{o}\left(\eta_{o}\right)+\beta_{c}\left(\eta_{c}\right)\right]$.

At jump instants, it is seen that only the variables $z_{d}$ and $y_{d}$ are reset which are not part of the definition of $V$. Hence, $V^{+}=V^{-}$at all time instants where the state jumps. Using this jump equation for $V$, and the bound derived for $\dot{V}$, we next show that the origin of the closed-loop system is GAS in following three steps:

Step 1 - Pre-GAS of $\{0\}$ for truncated systems: For a fixed initial condition, there exists a compact set $\mathcal{M}_{c}$ such that $\left(x, z, \eta_{o}, \eta_{c}\right) \in \mathcal{M}_{c}$. Recalling that $z_{d}$ and $y_{d}$ remain constant during flows, and are reset to $z$ and $h(x)$, which belong to a compact set, there exists a compact set $\mathcal{M}_{d}$ such that $\left(z_{d}, y_{d}\right) \in \mathcal{M}_{d}$. Consider the truncation of system (11) to the set $\mathcal{M}:=\mathbb{R}^{2 n+2} \times \mathcal{M}_{d}$, which has the flow set $\mathcal{C}_{\mathcal{M}}:=\mathcal{C} \cap$ $\mathcal{M}$, the jump set $\mathcal{D}_{\mathcal{M}}:=\mathcal{D}_{\mathcal{M}} \cap \mathcal{M}$. For this truncated system, it follows from the invariance principle [9, Corollary 8.9 (ii)] that the set $\mathcal{A}_{1}:=\left\{\bar{x}:\left(x, z, \eta_{o}, \eta_{c}\right)=\{0\} \wedge\left(y_{d}, z_{d}\right) \in\right.$ $\left.\mathcal{M}_{d}\right\}$ is pre-GAS. We next invoke the stability result for cascaded hybrid systems [8, Corollary 19] to claim that the set $\mathcal{A}_{2}:=\{0\} \in \mathbb{R}^{\bar{n}}$ is pre-GAS for the truncated system. Indeed, for every system trajectory contained in $\mathcal{A}_{1}$, we have $\eta_{o}=\eta_{c}=0$, and from the definition of the sets $\mathcal{C}$ and $\mathcal{D}$, we must then have $z_{d}=0$ and $y_{d}=0$.

Step 2 - Bounded solutions and Pre-GAS of $\{0\}$ for (11): As shown in the first step, for each initial condition, there exist compact sets $\mathcal{M}_{c}$ and $\mathcal{M}_{d}$ such that $\bar{x}$ is contained in the compact set $\mathcal{M}_{c} \times \mathcal{M}_{d}$ for all times. Boundedness of the solutions now allows us to conclude that $\mathcal{A}_{2}=\{0\}$ is preGAS for the original system (11). To see this, assume that there exists a solution which does not converge to $\{0\}$. Since all solutions are bounded, there exists a compact set $\mathcal{M}_{d}$ such that this bounded solution eventually coincides with the solution of the system truncated to $\mathbb{R}^{2 n+2} \times \mathcal{M}_{d}$. But, every solution of the truncated system must converge to $\{0\}$. 
Hence, for (11), a bounded solution not converging to $\{0\}$ cannot exist, proving that $\mathcal{A}_{2}=\{0\}$ is pre-GAS.

Step $3-\{0\}$ is GAS for (11): To move from preasymptotic stability to asymptotic stability of the compact set $\mathcal{A}_{2}=\{0\}$, we next show that every solution of (11) is forward complete. This is seen due to the fact that for each $\bar{x} \in \mathcal{C} \backslash \mathcal{D}$, the solutions would always continue to flow. Moreover, after each jump the states are reset to the set $\mathcal{C} \cup \mathcal{D}$, making it possible to extend the time domain for the solutions either by jump or flow. Hence, each solution of the system is forward complete, proving that the set $\mathcal{A}_{2}=\{0\}$ is GAS.

\section{EXISTENCE OF INTER-SAMPLING TIMES}

We next want to show that the proposed sampling algorithms given in Section III do not lead to the accumulation of jump times over a finite time interval, and over each compact interval, there exists a lower bound on inter-sampling times. Our strategy for showing the existence of minimal intersampling time is primarily based on the approach adopted in [23]. However, unlike [23], we do not get autonomous differential inequality that gives uniform lower bounds; instead, we obtain time-varying differential inequalities, and hence the inter-sampling times depend upon the interval under consideration.

For the proposed sampling routines, the minimum time between between two output (respectively, control input) updates is the time taken by $\frac{\left|y(t)-y_{d}(t)\right|}{\sigma_{o}\left(\eta_{o}(t)\right)}\left(\operatorname{resp} . \frac{\left|z(t)-z_{d}(t)\right|}{\sigma_{c}\left(\eta_{c}(t)\right)}\right)$ to go from 0 to 1 , after each time $y_{d}$ has been reset to current value of $y$ (resp. $z_{d}$ has been reset to $z$ ). In order to derive lower bounds on minimal time between updates, we will first introduce certain assumptions on the gain functions given in Section II and the ones used to define sampling instants in Section III.

\section{A. Assumptions on gain functions}

(A1) The system dynamics defined by $f, h$, and the controller functions $g, k$ are bounded by a linear growth rate, which allow us to write $\left|f\left(x, k\left(x+d_{e}\right)\right)\right| \leq L_{f k}(|x|+$ $\left.\left|d_{e}\right|\right)$ and $\left|g\left(z, k\left(z+d_{z}\right), h(x)+d_{y}\right)\right| \leq L_{g k}\left(|z|+\left|d_{z}\right|\right)+$ $L_{g h}\left(|x|+d_{y}\right)$. Also, $\|\partial h / \partial x\|$ is bounded by a constant.

(A2) The functions $\alpha_{o}$ and $\alpha_{c}$ are linear, and $\alpha_{o}(r)<\alpha_{c}(r)$.

(A3) The function $\gamma_{c} \circ \underline{\alpha}_{o}^{-1}$ is bounded by a linear growth rate: There exists $L_{c o}>0$ such that $\gamma_{c}\left(\underline{\alpha}_{o}^{-1}(r)\right) \leq L_{c o} r$.

(A4) The functions $\sigma_{o}, \sigma_{c}$ are same up to multiplication by a constant $C>0$, that is, $\sigma_{o}=C \sigma_{c}$. Furthermore, let $\sigma:=\min \left\{\sigma_{o}, \sigma_{c}\right\}$, and assume there are constants $C_{\sigma, 1}, C_{\sigma, 2}>0$, that satisfy

$$
\begin{gathered}
\sigma(r) \geq C_{\sigma, 1} \max \left\{\underline{\alpha}_{c}^{-1}(r), \underline{\alpha}_{o}^{-1}(r)\right\} \\
\sigma^{\prime}(r) \cdot r \leq C_{\sigma, 2} \sigma(r) .
\end{gathered}
$$

In addition, there exists a continuous locally integrable function $\chi: \mathbb{R}_{\geq 0} \rightarrow \mathbb{R}_{\geq 0}$ such that for every $r, s \geq 0$

$$
\frac{\sigma(r)}{\sigma(s)} \leq \chi\left(\frac{r}{s}\right) .
$$

Remark 3 (How restrictive are $(\mathbf{A 1})$ - (A4)?): One typically requires $f, g$ to be locally Lipschitz for existence of solutions, which would ensure that the linear growth rate condition holds on every compact set. A global linear bound (which is satisfied for globally Lipschitz functions) has been introduced to avoid the semi-global arguments in this article. For (A2), note that it is always possible to modify the Lyapunov functions $V_{o}, V_{c}$ so that the dissipation functions $\alpha_{o}$ and $\alpha_{c}$ are linear, see [18, Lemma 12]. However, this would also modify the gain function $\gamma_{c}$ and one must be careful in verifying hypothesis (H4). The most restrictive aspect of our approach is to verify (A4): As one would usually observe in the linear case, if $\underline{\alpha}_{c}(r)=\underline{\alpha}_{o}(r)=r^{2}$, then one can choose $\sigma(r)=\sqrt{r}$ (modulo multiplication with certain constants). In general, having $\sigma(r)=r^{\alpha}$, for $\alpha>0$, would satisfy (13b) and (13c). To find a constructive proof for existence of such $\sigma$ is a topic of ongoing work.

In the light of these assumptions, we now impose the following additional criteria on the design functions introduced in (6a), (6b), (7) and (8):

(D4) The functions $\beta_{o}, \beta_{c}$ are linear and for each $r \geq 0$

$$
\beta_{o}(r) \leq \alpha_{o}(r)<\beta_{c}(r) \leq \alpha_{c}(r) .
$$

(D5) The functions $\rho_{o}$ and $\rho_{c}$ are chosen such that

$$
\begin{aligned}
& \max \left\{\rho_{o} \circ \underline{\alpha}_{c}^{-1}(r), \rho_{o} \circ \underline{\alpha}_{o}^{-1}(r)\right\} \leq C_{\rho} r \\
& \max \left\{\rho_{c} \circ \underline{\alpha}_{c}^{-1}(r), \rho_{c} \circ \underline{\alpha}_{o}^{-1}(r)\right\} \leq C_{\rho} r
\end{aligned}
$$

for some constants $C_{\rho}>0$.

The main result on Zeno-freeness now follows. The proof has been omitted due to space constraints.

Theorem 2: If, in addition to the hypotheses of Theorem 1, assumptions (A1) - (A4) hold and the functions $\beta_{o}, \beta_{c}, \rho_{o}, \rho_{c}$ satisfy (D4) and (D5), then there is no accumulation point of the sampling times for outputs and inputs over a compact interval.

\section{EXAMPLE}

In order to demonstrate our design, and observe practical feasibility of our algorithms, we take a nonlinear system system with globally Lipschitz vector field. The calculations carried out in this example would carry over to linear systems with very slight modification. Consider the system

$$
\begin{aligned}
& \dot{x}_{1}=x_{2}+0.25\left|x_{1}\right| \\
& \dot{x}_{2}=\operatorname{sat}\left(x_{1}\right)+u
\end{aligned}
$$

with $y=x_{1}$. The notation $\operatorname{sat}\left(x_{1}\right)$ denotes the saturation function, that is, $\operatorname{sat}\left(x_{1}\right)=\min \left\{1, \max \left\{-1, x_{1}\right\}\right\}$. The nominal output feedback controller is:

$$
\begin{aligned}
\dot{z}_{1} & =z_{2}+0.25|y|+l_{1}\left(y-z_{1}\right) \\
\dot{z}_{2} & =\operatorname{sat}(y)+u+l_{2}\left(y-z_{1}\right) \\
u & =k(z)=\operatorname{sat}\left(z_{1}\right)-k_{1} z_{1}-k_{2} z_{2}
\end{aligned}
$$

where we pick $L^{\top}:=\left[\begin{array}{ll}l_{1} & l_{2}\end{array}\right]=\left[\begin{array}{ll}2 & 2\end{array}\right]$, and $K:=\left[\begin{array}{ll}k_{1} & k_{2}\end{array}\right]=$ $L^{\top}$. By choosing, $V_{o}(e)=e^{\top} P_{o} e$ and $V_{c}(x)=x^{\top} P_{c} x$, with $P_{o}=\left[\begin{array}{cc}2 & -1 \\ -1 & 1\end{array}\right]$ and $P_{c}=\left[\begin{array}{cc}1 & 0.5 \\ 0.5 & 0.5\end{array}\right]$, hypotheses (H2) and (H3) hold. Indeed, if the controller is driven by the sampled 

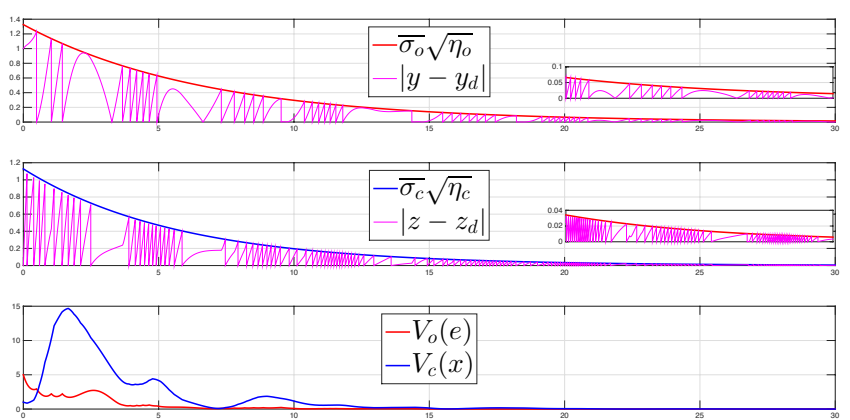

Fig. 2. Simulation results: In the top plot, whenever $\left|y(t)-y_{d}(t)\right|$ reaches the sampling threshold $\bar{\sigma}_{o} \sqrt{\eta_{o}(t)}, y_{d}$ is updated. The middle plot shows $\bar{\sigma}_{c} \sqrt{\eta_{c}(t)}$ and $\left|z(t)-z_{d}(t)\right|$. The bottom plot shows time evaluation of Lyapunov functions $V_{o}(e)$ and $V_{c}(x)$ which are not always decaying.

output $y_{d}$, then

$$
\dot{V}_{o} \leq-\alpha_{o} V_{o}+\bar{\gamma}_{o}\left|y_{d}-y\right|^{2}
$$

where $\bar{\gamma}_{o}:=\frac{\left\|P_{o} L\right\|^{2}}{p_{o} \lambda_{\min }\left(P_{o}\right)}$, and $\alpha_{o}+p_{o}=2$. Similarly, with $u_{d}=k\left(z_{d}\right)$, the derivative of $V_{c}(x)=x^{\top} P_{c} x$ satisfies

$$
\dot{V}_{c} \leq-\alpha_{c} V_{c}+\bar{\gamma}_{c}\left|z_{d}-z\right|^{2}+\bar{\gamma}_{c}|e|^{2}
$$

where $\bar{\gamma}_{c}:=\frac{2\left\|P_{c} B K\right\|^{2}}{p_{c} \lambda_{\min }\left(P_{c}\right)}$, and $\alpha_{c}+p_{c}=2-\|P\|$.

For sampling algorithms, we can let $q(s)=\bar{q}:=$ $\frac{4 \bar{\gamma}_{c}}{\alpha_{o} \lambda_{\min }\left(P_{o}\right)}$ to be the constant function and consider the following dynamic filters which satisfy the design criteria (D1) - (D3):

$$
\begin{aligned}
& \dot{\eta}_{o}=-\alpha_{o} \eta_{o}+\bar{\rho}_{o}|y(t)|^{2}+\bar{\gamma}_{o}\left|y-y_{d}\right|^{2} \\
& \dot{\eta}_{c}=-\alpha_{c} \eta_{c}+\bar{\rho}_{c} \frac{|z|^{2}}{4}+\bar{\gamma}_{c}\left|z-z_{d}\right|^{2}
\end{aligned}
$$

where, in the notation of (6), we have chosen $\beta_{o}(r)=$ $\alpha_{o} r$ and $\beta_{c}(r)=\alpha_{c} r$ for simplicity. Also, we let $\bar{\rho}_{o}:=$ $\frac{(1-2 \varepsilon) \lambda_{\min }\left(P_{o}\right)}{\|C\|^{2}}$ and $\bar{\rho}_{c}:=\min \left\{(1-\varepsilon) \bar{\gamma}_{c}, \varepsilon \alpha_{c} \lambda_{\min }\left(P_{c}\right)\right\}$. The jump sets for the closed-loop system which determine the sampling times are now defined as follows:

$$
\begin{aligned}
& \mathcal{D}_{o}=\left\{\bar{x}:\left|h(x)-y_{d}\right| \geq \bar{\sigma}_{o} \sqrt{\eta_{o}}\right\} \\
& \mathcal{D}_{c}=\left\{\bar{x}:\left|z-z_{d}\right| \geq \bar{\sigma}_{c} \sqrt{\eta_{c}}\right\}
\end{aligned}
$$

where $\bar{\sigma}_{o}:=\frac{(1-\varepsilon) \alpha_{o}}{(1+\bar{q}) \bar{\gamma}_{o}}$, and $\bar{\sigma}_{c}=\frac{(1-\varepsilon) \alpha_{c}}{2 \bar{\gamma}_{c}}$. From Theorem 1, asymptotic stability of the closed-loop system with sampled outputs and inputs now follows. It can also be verified that (A1) - (A4) hold by construction.

The results of the simulation appear in Figure 2. We observed that, even though the constants $\bar{\sigma}_{o}$ and $\bar{\sigma}_{c}$ are relatively small in magnitude, it was possible to slow down the sampling rate by increasing the initial values of $\eta_{o}$, and $\eta_{c}$. Also, the Lyapunov functions $V_{o}(e)$ and $V_{c}(x)$ are not always decaying but the function $V$ in (12) is indeed decaying with time.

\section{CONCLUSIONS}

We have proposed novel type of sampling algorithms which use the state-variables of auxiliary designed dynamical systems. If an output feedback control law which has certain robustness properties in the ISS sense, then the proposed sampling algorithms preserve the stability of the closed-loop system. Under added assumptions on the gains of the ISS estimate, the proposed event-triggered schemes do not lead to an accumulation of sampling times over a finite interval which makes it feasible from implementation point of view.

\section{REFERENCES}

[1] M. Abdelrahim, R. Postoyan, J. Daafouz and D. Nesic. Stabilization of nonlinear systems using event-triggered output feedback laws. In Proc. Int. Symp. Math. Theory of Networks and Systems, Gronigen, The Netherlands, 2014.

[2] V. Andrieu and L. Praly. A unifying point of view on output feedback designs for global asymptotic stabilization. Automatica, 45(8): 1789 $-1798,2009$.

[3] A. Anta and P. Tabuada. To sample or not to sample: self-triggered control for nonlinear systems. IEEE Trans. Automatic Control, 55(9):2030 - 2042, 2010.

[4] K. Astrom. Event based control. In Analysis and Design of Nonlinear Control Systems, pages 127 - 148. Springer, 2008.

[5] V.S. Dolk, D.P. Borgers and W.P.M.H. Heemels. Dynamic eventtriggered control: Tradeoffs between transmission intervals and performance. In Proc. IEEE Conf. Decision \& Control, 2014.

[6] M.C.F. Donkers and W.P.M.H. Heemels. Output-based event-triggered control with guaranteed $\mathcal{L}_{\infty}$-gain and improved and decentralized event-triggering. IEEE Trans. Automatic Control, 57(6):1362 - 1376, 2012.

[7] A. Girard. Dynamic event generators for event-triggered control systems. IEEE Trans. Automatic Control, 60(7):1992 - 1997, 2015.

[8] R. Goebel, R. Sanfelice, and A. Teel. Hybrid dynamical systems. IEEE Control Sys. Magazine, 28-93, 2009.

[9] R. Goebel, R. Sanfelice, and A. Teel. Hybrid Dynamical Systems: Modeling, Stability and Robustness. Princeton University Press, 2012.

[10] W.P.M.H. Heemels, M.C.F. Donkers. Model-based periodic eventtriggered control for linear systems. Automatica, 49:698 - 711, 2013.

[11] W.P.M.H. Heemels, M.C.F. Donkers and A. Teel. Periodic eventtriggered control for linear systems. IEEE Trans. Automatic Control, 58(4):847 - 861, 2013.

[12] W.P.M.H. Heemels, K.H. Johansson, and P. Tabuada. An introduction to event-triggered and self-triggered control. In Proc. 51st IEEE Conf. Decision \& Control, Maui (HI), pp. 3270 - 3285, 2012.

[13] D. Lehmann and J. Lunze. Event-based output-feedback control. In Proc. 19th Mediterranean Conf. on Control and Automation, pages 982 - 987, 2011

[14] J. Lunze and D. Lehmann. A state-feedback approach to event-based control. Automatica, pages $211-215,2010$.

[15] D. Nesic and A. R. Teel. A framework for stabilization of nonlinear sampled-data systems based on their approximate discrete-time models. IEEE Trans. Automatic Control, 49(7): 1103 - 1122, 2004.

[16] D. Nesic and A. R. Teel. Input-output stability properties of networked control systems. IEEE Trans. Automatic Control, 49(10): 1654 - 1667, 2004.

[17] R. Postoyan, A. Anta, D. Nesic, and P. Tabuada. A unifying Lyapunovbased framework for the event triggered control of nonlinear systems. In Proc. Joint 50th IEEE Conf. Decision \& Control and European Control Conf., Orlando (FL), pp. 2559 - 2564, 2011.

[18] L. Praly and Y. Wang. Stabilization in spite of matched unmodeled dynamics and an equivalent definition of input-to-state stability. Math. Controls, Signals \& Sys., 9:1 - 33, 1996.

[19] A. Seuret, C. Prieur, and N. Marchand. Stability of nonlinear systems by means of event-triggered sampling algorithms. IMA J. of Math. Control and Information, 31(3): 415 - 433, 2014.

[20] H. Shim and D. Liberzon. Nonlinear observers robust to measurement disturbances in an ISS sense. IEEE Trans. on Automatic Control, to appear, 2015.

[21] E. Sontag and A. Teel. Changing supply functions in input/state stable systems. IEEE Trans. Automatic Control, 40(8): 1476 - 1478, 1995.

[22] W. Sontag and Y. Wang. Output-to-state stability and detectability of nonlinear systems. Sys. \& Control Letters, 29: 279 - 290, 1997.

[23] P. Tabuada. Event-triggered real-time scheduling of stabilizing control tasks. IEEE Trans. Automatic Control, 52(9):1680 - 1685, 2007.

[24] P. Tallapragada and N. Chopra. Event-triggered dynamic output feedback control for LTI systems. In Proc. 51st IEEE Conf. on Decision and Control, pages 6597 - 6602, 2012.

[25] A. Teel and L. Praly. Tools for semiglobal stabilization by partial state and output feedback. SIAM J. Control \& Optimization, 33(5): 1443 1488, 1995. 